\title{
Spatially Correlated Single Molecule Spectroscopy and Scanning Probe Microscopy for Characterizing Aggregation of Semiconductor Nanocrystals and Biomolecules
}

\author{
Alan Van Orden, Douglas Shepherd, and Ming Yu \\ Department of Chemistry, Colorado State University, Fort Collins, CO 80523
}

This paper describes a technique for performing spatially correlated single molecule fluorescence spectroscopy and atomic force microscopy [1]. Figure 1 displays a schematic of the instrumentation. The purpose of this technique is to characterize the relationship between the fluorescence emission dynamics and the nanometer scale topography of individual nanoparticles, biomolecules, and molecular scale clusters and complexes immobilized on surfaces. The information provided can shed important light on the spectroscopic and structural properties of a wide range of systems.

The present paper will discuss the application of this technique to the study of individual, isolated semiconductor nanocrystals and small ordered clusters of the nanocrystals. Applications of these systems in the areas of solar photovoltaics and biological sensing and imaging will also be discussed. Single molecule fluorescence measurements reveal that individual semiconductor nanocrystals undergo fluorescence intemittency, or "blinking," whereby their fluorescence emission fluctuates between "on" and "off” states [2]. Our research has shown that when multiple nanocrystals are probed simultaneously, the blinking dynamics depends on the distance between nanocrystals. In samples of isolated nanocrystals, the observed blinking is characteristic of multiple nanocrystals blinking independently. However, when small aggregates of nanocrystals are formed, the blinking becomes cooperative (See Figure 2) [3].

This cooperative blinking has applications in solar photovoltaics and in biological sensing and imaging. For example, we will discuss the interactions of semiconductor nanocrystal clusters with single crystal $\mathrm{TiO}_{2}$ surfaces. It has been found that the nanocrystals interact with $\mathrm{TiO}_{2}$ such that injection and trapping of photoexcited charge carriers alter the blinking behavior of the nanocrystals. Understanding the mechanism for these interactions can lead to improved performance of nanocrystalline sensitized solar photovoltaic cells.

Additionally, the altered blinking of individual nanocrystals is being used to characterize the dynamics and aggregation of cell surface receptors. The nanocrystals are being used as fluorescent labels for the receptors, and fluorescence correlation spectroscopy is being used to characterize the motion of the nanocrystals. Aggregate formation is characterized based on the change in the cooperative blinking that occurs when the nanocrystals form small ordered clusters.

\section{References}

[1] L. Kolodny, et al., Anal. Chem. 73 (2001) 1959.

[2] M. Kuno, et al., Phys. Rev. B 67 (2003) 125304.

[3] M. Yu and A. Van Orden, Phys. Rev. Lett. 97 (2006) 237402.

[4] This research is supported by the Colorado Renewable Energy Collaboratory-Center for Revolutionary Solar Photoconversion and the NSF Collaborative Research in Chemistry Program (Grant 0628260). 
Figure 1. The CSU spatially correlated single molecule fluorescence / AFM apparatus. PD: Analog photodiodes; APD: Single photon counting modules. The emission optics are configured for wavelength resolved detection. Analysis of polarized emission is also possible.
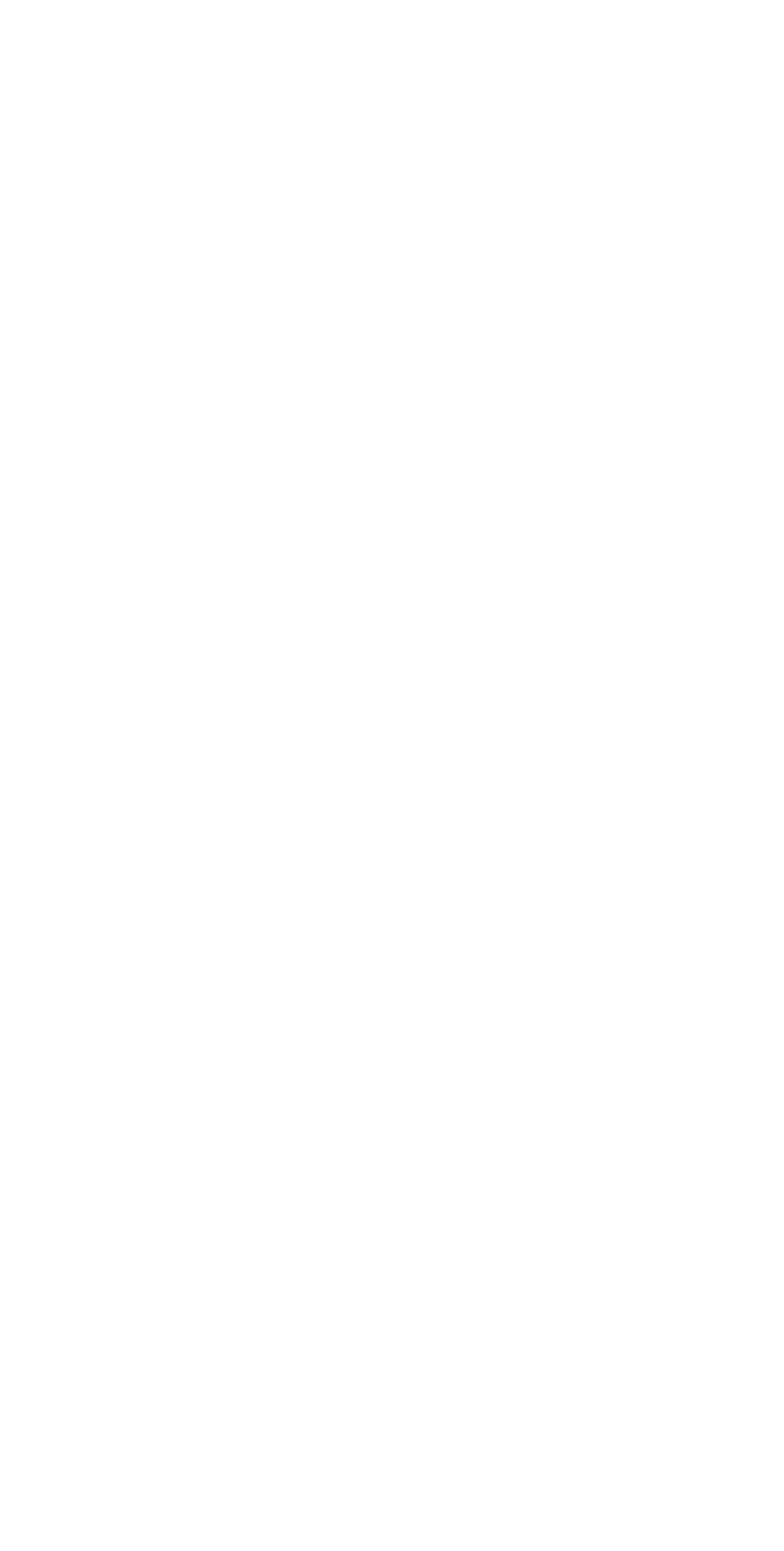

Figure 2. Single molecule fluorescence trajectories (left), photon count histograms (right), and AFM topography images (inset) of (A) a single isolated CdSeZnS nanocrystal, (B) a cluster of CdSe-ZnS nanocrystals, (C) three isolated nanocrystals probed simultaneously, and (D) multiple isolated nanocrystals probed simultaneously . The AFM images have scale bars of 98-nm and z-ranges of 8nm (Reprinted from Ref. 3, Copyright American Physical Society). 